\title{
Synthesis and structure of a new layer compound: $\mathrm{ScPS}_{4}$
}

\author{
Stephen Lee* and Robert J. Hilt \\ Department of Chemistry, University of Michigan, Ann Arbor, MI 48109-1055 (USA)
}

(Received January 30, 1992; in final form June 17, 1992)

\begin{abstract}
$\mathrm{ScPS}_{4}$, which belongs to a new structure type, was prepared and characterized by single crystal X-ray diffraction. It has a layer structure with seven-coordinate scandium. The space group is $P \overline{1}$ and the lattice constants are $a=6.445(5) \AA, b=6.659(5) \AA, c=6.211(4) \AA, \alpha=91.36(5), \beta=98.76(5)$, and $\gamma=86.22(5)$. The final $R / R_{w}$ for the crystal refinement was $4.9 / 5.1 \%$.
\end{abstract}

\section{Introduction}

In recent years there has been a marked and widespread interest in two-dimensional materials [1]. Such materials display interesting properties such as charge density waves and high $T_{\mathrm{c}}$ superconductivity. They also form the templates for further reactions as is well known in intercalation chemistry [2]. We report in this article the synthesis of what we believe is a new structure type for a two-dimensional material. This material is $\mathrm{ScPS}_{4}$. It belongs to the family of compounds, $\mathrm{MPS}_{4}$ $(\mathrm{M} \equiv \mathrm{B}, \mathrm{Al}, \mathrm{Ga}$, In, $\mathrm{Bi}$, and $\mathrm{Cr})[3]$ where $\mathrm{M}$ is a +3 oxidation-state metal.

\section{Experimental details}

Crystals of $\mathrm{ScPS}_{4}$ were prepared from the elements (purchased from Alfa Products with nominal purities: Sc 99.9\%, - 40 mesh; P 99\%, - 100 mesh; and S $99.999 \%$, flakes) by vapor transport in quartz tubes between $690^{\circ} \mathrm{C}$ and $670^{\circ} \mathrm{C}$. The crystals are light green and form at the cool end as platelets which readily break into thin fibers. The phase is air sensitive; the powder decomposes in a few minutes while larger single crystals are somewhat less prone to attack. Crystals were therefore selected in an inert atmosphere glove box and mounted in sealed capillaries. After reviewing the crystals on a Weissenberg camera, the best crystal was placed on a $\mathbf{P} 2$, Syntex four-circle diffractometer. The best crystals proved to be oversized with respect to the X-ray beam. However, attempts to cut them proved unsuccessful owing to their fragile nature. A

*Author to whom correspondence should be addressed. summary of the conditions of the crystal structure determination is given in Table 1 . The structure was

TABLE 1. Summary of crystallographic data for ScPS 4 . Data collected at room temperature. Secondary extinction correction and absorption corrections using crystal shape were made

\begin{tabular}{ll}
\hline Chemical formula & $\mathrm{ScPS}_{4}$ \\
Space group & $P \overline{1}$ \\
$a$ & $6.445 \AA$ \\
$b$ & $6.659 \AA$ \\
$c$ & $6.211 \AA$ \\
$\alpha$ & $91.36^{\circ}(5)$ \\
$\beta$ & $98.76^{\circ}(5)$ \\
$\gamma$ & $86.22^{\circ}(5)$ \\
$V$ & $262.8(4) \AA^{3}$ \\
$Z$ & 2 \\
Radiation & $\lambda(\mathrm{Mo} \mathrm{K \alpha})=0.71069 \AA$ \\
Absorption coefficient $(\mu)$ & $46.56 \mathrm{~cm}^{-1}$ \\
$2 \theta_{\text {max }}$ & $45^{\circ}$ \\
Reflections collected & 731 \\
Unique reflections & 686 \\
Reflections used in refinement & 662 \\
$\quad\left(F_{\mathrm{o}}{ }^{2}>3 \sigma\left(F_{\mathrm{o}}{ }^{2}\right)\right)$ & \\
Number of variables & 56 \\
$R, R_{w}$ & $4.9 \%, 5.1 \%$ \\
Crystal dimension & $0.17 \times 0.31 \times 1.7 \mathrm{~mm}^{3}$ \\
Color & pale green \\
Observed/parameter ratio & 12.3 \\
$R$ & $\left.\Sigma\left(\left|F_{\mathrm{o}}-F_{\mathrm{c}}\right|\right) / \Sigma\left|F_{\mathrm{o}}\right|\right)$ \\
$R_{w}$ & {$\left[\Sigma\left(\left|F_{\mathrm{o}}-F_{\mathrm{c}}\right|\right)^{2} / \Sigma w\left|F_{\mathrm{o}}\right|^{2}\right]^{1 / 2}$} \\
$w^{-1}$ & $\sigma^{2}\left(F_{\mathrm{o}}\right)$ \\
\hline
\end{tabular}

Anisotropically refined atoms are given in the form of the isotropic equivalent thermal parameter, $U(\mathrm{eq})$, defined as $4 / 3\left[a^{2} U_{11}+\right.$ $\left.b^{2} U_{22}+c^{2} U_{33}+a b(\cos \gamma) U_{12}+a c(\cos \beta) U_{13}+b c(\cos \alpha) U_{23}\right]$.

Scattering factors taken from J. A. Ibers and W. C. Hamilton (eds.), International Tables for Cnystallography, Vol. 4, Kynoch Press, Birmingham, 1974. 
TABLE 2. Fractional atomic parameters for $\mathrm{ScPS}_{4}{ }^{\mathrm{a}}$

\begin{tabular}{lllll}
\hline Atom & $X$ & $Y$ & $Z$ & $U_{\text {iso }}$ \\
\hline Sc & $0.3038(2)$ & $0.9345(2)$ & $0.2062(2)$ & 0.010 \\
P & $0.2640(3)$ & $0.8081(2)$ & $0.6896(3)$ & 0.009 \\
S $(1)$ & $0.0364(3)$ & $0.7838(3)$ & $0.8831(3)$ & 0.013 \\
S $(2)$ & $0.2825(3)$ & $0.0995(2)$ & $0.5967(3)$ & 0.011 \\
S(3) & $0.5274(3)$ & $0.7453(2)$ & $0.9150(3)$ & 0.010 \\
S(4) & $0.2361(3)$ & $0.6320(2)$ & $0.4218(3)$ & 0.014 \\
\hline
\end{tabular}

accupancy parameters were not refined.

TABLE 3. Bond distances for Sc and P in $\mathrm{ScPS}_{4}$

\begin{tabular}{lll}
\hline Atom & Atom & Distance $(\AA)$ \\
\hline Sc & $1 S(1)$ & $2.661(2)$ \\
& $1 S(1)$ & $2.788(2)$ \\
& $1 S(2)$ & $2.658(2)$ \\
& $1 S(2)$ & $2.757(2)$ \\
& $1 S(3)$ & $2.632(2)$ \\
& $1 S(3)$ & $2.717(2)$ \\
& $1 S(4)$ & $2.542(2)$ \\
$P$ & $1 S(1)$ & $2.049(2)$ \\
& $1 S(2)$ & $2.054(2)$ \\
& $1 S(3)$ & $2.056(2)$ \\
& $1 S(4)$ & $2.004(2)$ \\
\hline
\end{tabular}

initially solved by the SHELXs-86 package [4] which, upon least-squares refinement (using SHELX-77), resulted in the positional parameters given in Table 2. Bond distances are given in Table 3. The highest peak in the resulting difference Fourier map had a height of 0.77 e $\AA^{-3}$. The final $R / R_{w}$ factor was $4.9 / 5.1 \%$. After atomic positions were determined, a search for additional symmetry elements was undertaken. No such elements could be found, and we therefore conclude the structure is indeed in the $P \overline{1}$ space group. A Guinier powder (Enraf-Nonius camera) pattern was taken from a sample placed in a capillary. All lines present were indexable according to the refined single crystal structure as calculated by the LAZY-PULVERIX program [5].

\section{Discussion and results}

The overall connectivity in $\mathrm{ScPS}_{4}$ is that of twodimensional layer sheets. These sheets lie in crystallographic planes normal to the $b$ axis as shown in Fig. 1. As may be seen in the figure, the Sc atoms are seven-coordinate to sulfur. Bond distances range from $2.542 \AA$ to $2.788 \AA$. The overall Sc coordination polyhedra are slightly distorted monocapped trigonal prisms. Between these seven-coordinate scandium atoms are $\mathrm{PS}_{4}^{3-}$ tetrahedra, in keeping with the +3 oxidation state of the Sc atom. These units may be viewed in a manner so as to interrelate this structure with the structures of AlPS 4 and BPS 4 . In AlPS 4 and BPS 4 (as in all MPS ${ }_{4}$ compounds) there are also $\mathrm{PS}_{4}^{3-}$ tetrahedra. However, because of the smaller size of the $\mathrm{Al}$ and $\mathrm{B}$ atoms, these metal atoms are only four-coordinate to sulfur. The overall structural motif of both $\mathrm{AlPS}_{4}$ and $\mathrm{BPS}_{4}$ is that of edge-sharing chains of sulfur tetrahedra. These tetrahedra are occupied by $\mathrm{P}$ and $\mathrm{Al}$ or $\mathrm{B}$ cations. The occupation in the tetrahedral chains alternates systematically between the small group 13 metal atoms and $P$ atoms. This same structural unit may be seen in the $\mathrm{ScPS}_{4}$ structure. To see this we consider only four of the seven vertices of the monocapped trigonal prism which surrounds each sulfur. These four are
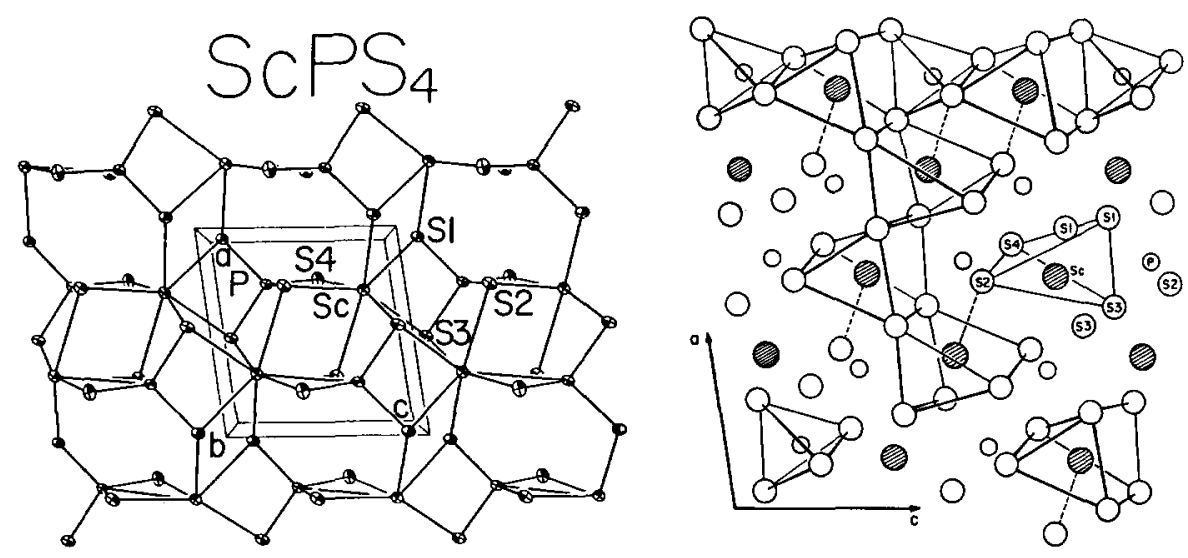

Fig. 1. A single sheet in the $\mathrm{ScPS}_{4}$ structure. We show here the $a-c$ plane on both sides. Left side: ORTEP drawing showing both the P-S and Sc-S bonds using 50\% thermal ellipsoids. Right side: the same sheet from a slightly different orientation. Striped circles are Sc atoms, large unfilled circles $S$ atoms and small circles $P$ atoms. The distorted tetrahedral chains of Fig. 2, run horizontally (along $c$ direction) (see top of figure). We show the full capped trigonal prismatic coordination of the Sc atoms. Running along the $a$ direction are chains of edge-sharing trigonal prisms. Together, the tetrahedral and trigonal prismatic chains form a twodimensional sheet. Also shown are the coordination polyhedra for both the P and Sc atoms. A view of the distorted Sc tetrahedra is also given. 


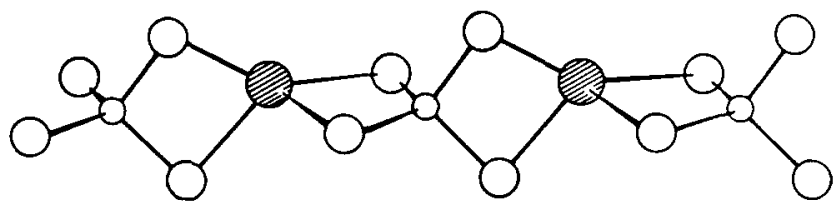

Fig. 2. The distorted tetrahedral chain found in $\mathrm{ScPS}_{4}$. Large striped circles are $\mathrm{Sc}$, small circles $\mathrm{P}$ and intermediate sized ones $S$. It may be seen that the $P$ atoms lie in the center of nearly perfect tetrahedra. By contrast the $\mathrm{Sc}$ atoms are very much distorted. Indeed, as Fig. 1 shows, Sc actually is seven-coordinate. Near-perfect tetrahedral chains are to be found in $\mathrm{AlPS}_{4}$ and $\mathrm{BPS}_{4}[3]$.

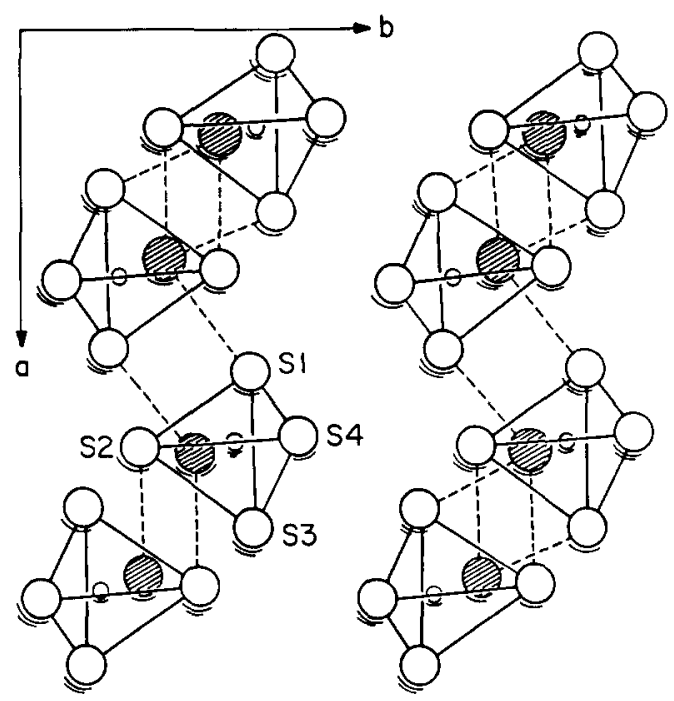

Fig. 3. The $\mathrm{ScPS}_{4}$ structure viewed along the $a-b$ plane. The distorted tetrahedral chains are now normal to the plane of the paper. The corrugated sheet structure of $\mathrm{ScPS}_{4}$ is clear. The van der Waals gap between layers is similarly corrugated.

shown in the center of the right side of Fig. 1. Considering only these four Sc-S bonds, one finds in $\mathrm{ScPS}_{4}$ chains of edge-sharing tetrahedra running along the $c$ crystal axis. We isolate one such structural unit in Fig. 2. As may be seen in this figure, the $\mathrm{ScPS}_{4}$ tetrahedra are quite distorted owing to the true seven-fold coordination of the scandium atoms. In Fig. 3 we show these chains in a head-on view. Figure 3 also shows clearly the van der Waals gap between the layers. This gap is corrugated in shape and is normal to the $b$ axis.
Supplementary material reporting anisotropic parameters and $F_{\mathrm{o}}$ and $F_{\mathrm{c}}$ can be obtained from the authors.

\section{Acknowledgments}

We thank the Rackham Graduate School for partial support of this work and Dr. William Butler for recording the data set on the $\mathrm{P} 2_{1}$ diffractometer. Partial support for this work came from the Petroleum Research Fund administered by the ACS.

\section{References}

1 F. Levy (ed.), Crystallography and Crystal Chemistry of Materials with Layered Structures, Vols. 1 and 2, Reidel, Dordrecht, 1976.

T. J. Wieting and M. Schlüter (eds.), Electrons and Phonons in Layered Crystal Structures, Reidel, Dordrecht, 1979.

V. Grasso (ed.), Electronic Structure and Electronic Transitions in Layered Materials, Reidel, Dordrecht, 1986.

Z. Gan and Z. Su, Two Dimensional Strongly Correlated Electronic Systems, Gordon and Breach, New York, 1989.

J. A. Wilson, F. J. DiSalvo and S. Mahajan, Adv. Phys., 24 (1975) 117.

J. Rouxel (ed.), Crystal Chemistry and Properties of Materials with Quasi-One-Dimensional Structures, Reidel, Dordrecht, 1986.

2 M. S. Whittingham and A. J. Jacobson, Intercalation Chemistry, Academic Press, New York, 1982.

J. Rouxel, Physica, $99 B$ (1980) 3.

J. Rouxel, in P. Barret and L. C. Dufour (eds.), Reactivity of Solids, Elsevier, Amsterdam, 1985.

3 A. Weiss and H. Schaefer, Z. Naturforsch., $18 b$ (1963) 81. A. Weiss and H. Schaefer, Naturwissenschaften, 47 (1960) 495.

P. Buck and C. D. Carpentier, Acta Crystallogr., B29 (1973) 1864.

H. Zimmermann, C. D. Carpentier and R. Nitsche, Acta Crystallogr., B31 (1975) 2003.

C. D. Carpentier, R. Diehl and R. Nitsche, Naturwissenschaften, 57 (1970) 393.

R. Diehl and C. D. Carpentier, Acta Crystallogr., B33 (1977) 1399.

4 G. M. Sheldrick, in G. M. Sheldrick, C. Krüger and R. Goddard (eds.), Crystallographic Computing 3, Oxford Press, Oxford, 1985, p. 175.

5 K. Yvon, W. Jeitschko and E. Parthé, J. Appl. Crystallogr., 10 (1977) 73. 